\title{
Once-weekly albiglutide versus once-daily liraglutide in patients with type 2 diabetes inadequately controlled on oral drugs (HARMONY 7): a randomised, open-label, multicentre, non-inferiority phase 3 study
}

Richard E Pratley, Michael A Nauck, Anthony H Barnett, Mark N Feinglos, Fernando Ovalle, Illana Harman-Boehm, June Ye, Rhona Scott, Susan Johnson, Murray Stewart, Julio Rosenstock, for the HARMONY 7 study group

\section{Summary}

Background As new members of a drug class are developed, head-to-head trials are an important strategy to guide personalised treatment decisions. We assessed two glucagon-like peptide-1 receptor agonists, once-weekly albiglutide and once-daily liraglutide, in patients with type 2 diabetes inadequately controlled on oral antidiabetic drugs.

Methods We undertook this 32-week, open-label, phase 3 non-inferiority study at 162 sites in eight countries: USA (121 sites), Australia (9 sites), Peru (7 sites), Philippines (7 sites), South Korea (5 sites), UK (5 sites), Israel (4 sites), and Spain (4 sites). 841 adult participants (aged $\geq 18$ years) with inadequately controlled type 2 diabetes and a BMI between 20 and $45 \mathrm{~kg} / \mathrm{m}^{2}$ were enrolled and randomised in a 1:1 ratio to receive albiglutide $30 \mathrm{mg}$ once weekly titrated to $50 \mathrm{mg}$ at week 6 , or liraglutide $0.6 \mathrm{mg}$ once daily titrated to $1.2 \mathrm{mg}$ at week 1 and $1.8 \mathrm{mg}$ at week 2 . The randomisation schedule was generated by an independent randomisation team by the permuted block method with a fixed block size of 16. Participants and investigators were unmasked to treatment. The primary endpoint was change from baseline in $\mathrm{HbA}_{1 \mathrm{c}}$ for albiglutide versus liraglutide, with a 95\% CI non-inferiority upper margin of $0 \cdot 3 \%$. The primary analysis was by modified intention to treat. The study is registered with ClinicalTrials.gov, number NCT01128894.

Findings 422 patients were randomly allocated to the albigultide group and 419 to the liraglutide group; 404 patients in the abliglutide group and 408 in the liraglutide group received the study drugs. The primary endpoint analysis was done on the modified intention-to-treat population, which included 402 participants in the albiglutide group and 403 in the liraglutide group. Model-adjusted change in $\mathrm{HbA}_{1 \mathrm{c}}$ from baseline to week 32 was $-\mathbf{0} \cdot 78 \%$ (95\% CI -0.87 to $-0.69)$ in the albigludite group and $-0.99 \%(-1.08$ to -0.90$)$ in the liraglutide group; treatment difference was $0 \cdot 21 \%$ $(0 \cdot 08-0 \cdot 34$; non-inferiority $p$ value $=0 \cdot 0846)$. Injection-site reactions occurred in more patients given albiglutide than in those given liraglutide $(12 \cdot 9 \%$ vs $5.4 \%$; treatment difference $7 \cdot 5 \%$ [95\% CI 3.6-11.4]; p=0 0002), whereas the opposite was the case for gastrointestinal events, which occurred in $49.0 \%$ of patients in the liraglutide group versus $35.9 \%$ in the albiglutide group (treatment difference $-13 \cdot 1 \%$ [95\% CI $-19 \cdot 9$ to $-6 \cdot 4]$; $\mathrm{p}=0 \cdot 00013$ ).

Interpretation Patients who received once-daily liraglutide had greater reductions in $\mathrm{HbA}_{1 \mathrm{c}}$ than did those who received once-weekly albiglutide. Participants in the albiglutide group had more injection-site reactions and fewer gastrointestinal events than did those in the liraglutide group.

Funding GlaxoSmithKline.

\section{Introduction}

Clinical studies have consistently shown that injectable glucagon-like peptide-1 (GLP-1) receptor agonists improve diabetes control by promoting glucosedependent insulin secretion, reducing postprandial glucagon secretion, and inducing satiety and weight loss. ${ }^{1,2}$ Liraglutide is a once-daily GLP-1 receptor agonist that is acylated to prolong its half-life. ${ }^{3}$ Albiglutide is a GLP-1 receptor agonist composed of a dipeptidyl peptidase 4-resistant GLP-1 dimer fused to recombinant human albumin, with a half-life of roughly 5 days..$^{47}$ In a previous phase 2 trial, albiglutide reduced $\mathrm{HbA}_{1 \mathrm{c}}$ at doses of $30 \mathrm{mg}$ weekly $(-0 \cdot 87 \%$ [SD $0 \cdot 65]), 50 \mathrm{mg}$ every 2 weeks $(-0 \cdot 79 \%$ $[0 \cdot 98])$, and $100 \mathrm{mg}$ monthly $(-0 \cdot 87 \%$ [0.87]) compared with placebo. ${ }^{5}$ The lowest frequency of gastrointestinal adverse events was reported with the $30 \mathrm{mg}$ weekly dose. ${ }^{5}$ In this head-to-head phase 3 trial, we compare the efficacy and safety of once-weekly albiglutide versus once-daily liraglutide in patients with type 2 diabetes uncontrolled on their existing oral antidiabetic treatment to provide objective evidence about the benefits and limitations of these two members of the GLP-1 receptor agonist drug class.

\section{Methods}

Study design and participants

Harmony 7 was a randomised, open-label, parallel-group, multicentre, phase 3 study, with the aim of assessing the
Lancet Diabetes Endocrino 2014; 2: 289-97 Published Online February 6, 2014 http://dx.doi.org/10.1016 S2213-8587(13)70214-6

This online publication has been corrected. The corrected version first appeared at thelancet.com/ diabetes-endocrinology on February 18, 2014

See Comment page 266

Florida Hospital Diabetes and Translational Research Institute, Sanford-Burnham Medical Research Institute, Orlando, FL, USA (R E Pratley MD); Diabeteszentrum Bad Lauterberg, Harz, Germany (Prof M A Nauck MD); Birmingham Heartlands Hospital, Birmingham, UK (Prof A H Barnett MD); Duke University, Durham, NC, USA (M N Feinglos MD); University of Alabama at Birmingham School of Medicine, Birmingham, AL, USA (F Ovalle MD); Ben-Gurion University of the Negev, Beer Sheva, Israel

(I Harman-Boehm MD); GlaxoSmithKline, Research Triangle Park, NC, USA (J Ye PhD, SJohnson MD); GlaxoSmithKline, Stevenage, Hertfordshire, UK (R Scott BSc[Hons]); GlaxoSmithKline, Upper Merion, PA, USA (M Stewart DM); and Dallas Diabetes and Endocrine Center, Dallas, TX, USA (J Rosenstock MD) Correspondence to: Dr Richard E Pratley, Florida Hospital Diabetes and Translational Research Institute Sanford-Burnham Medical Research Institute, 2415 North Orange Avenue, Orlando, FL 32804, USA richard.pratley@flhosp.org 
efficacy and safety of once-weekly albiglutide versus once-daily liraglutide in patients with type 2 diabetes uncontrolled on oral antidiabetic drugs. The study comprised four periods: screening, 4 weeks of run-in and stabilisation, 32 weeks of treatment, and 8 weeks of post-treatment follow-up. Data were collected from participants between May 5, 2010 (first participant, first visit), and Sept 9, 2011 (last participant, last visit).

Enrolment of participants began on April 19, 2010, and was completed on Oct 22, 2010. Enrolled patients were at least 18 years old, with type 2 diabetes uncontrolled $\left(\mathrm{HbA}_{1 \mathrm{c}}\right.$ $\geq 7.0 \%$ and $\leq 10 \cdot 0 \%$ ) on metformin, thiazolidinediones, sulfonylureas, or any combination of these drugs, and a BMI of at least $20 \mathrm{~kg} / \mathrm{m}^{2}$ but no higher than $45 \mathrm{~kg} / \mathrm{m}^{2}$. Patients were recruited from 162 sites in eight countries: the USA (121 sites, $n=643$ ), Australia ( 9 sites, $n=33$ ), Peru (7 sites, $\mathrm{n}=67)$, the Philippines ( 7 sites, $\mathrm{n}=25$ ), South Korea ( 5 sites, $\mathrm{n}=35$ ), the UK ( 5 sites, $\mathrm{n}=10$ ), Israel (4 sites, $\mathrm{n}=16$ ), and Spain (4 sites, $\mathrm{n}=12$ ). Most of the sites were clinics. Key exclusion criteria included history of type 1 diabetes, lipase concentration higher than the upper limit of normal, and cardiovascular event within the previous 2 months, cerebrovascular event within the previous month, or both (full inclusion and exclusion criteria in appendix pp 1-6).

The study was done in accordance with all applicable regulatory requirements, good clinical practice, all applicable patient privacy requirements, and the guiding principles of the Declaration of Helsinki. The study protocol, any amendments, the informed consent, and other information that required pre-approval were reviewed and approved by a national, regional, or investigational centre ethics committee or institutional review board, in accordance with the International Conference on Harmonisation of Technical Requirements for Registration of Pharmaceuticals for Human Use Good Clinical Practice and applicable country-specific requirements. All patients provided written, informed consent.

\section{Randomisation and masking}

The randomisation sequence was generated by an independent randomisation team, with the permuted block method with a fixed block size of 16 . On the basis of a sequestered fixed randomisation schedule, we randomly assigned roughly 400 patients to each of the two treatment groups in a 1:1 ratio. Eligible participants were stratified by $\mathrm{HbA}_{1 \mathrm{c}}$ value at week $-1 \quad<8 \cdot 0 \%$ vs $\geq 8 \cdot 0 \%$ ), previous history of myocardial infarction (yes or no), and age ( $<65$ years vs $\geq 65$ years). We randomly assigned treatment using an interactive voice recognition system. The study was open-label, so patients and investigators were unmasked to treatment.

\section{Procedures}

Eligible participants were randomly assigned to receive subcutaneous albiglutide $30 \mathrm{mg}$ weekly with uptitration to $50 \mathrm{mg}$ at week 6 , or subcutaneous daily liraglutide $0.6 \mathrm{mg}$ in week $1,1.2 \mathrm{mg}$ in week 2 , and $1.8 \mathrm{mg}$ thereafter. Patients self-injected the study drugs, which were dispensed at baseline. Patients returned all unused vials and used pens to establish adherence.

Patients who had hyperglycaemia during the study could be given rescue treatment at any time on or after week 2 according to predefined criteria (appendix p 7). The preferred add-on rescue treatment was insulin, and other treatments could be added at the investigator's discretion; however, the addition of another GLP-1 receptor agonist was prohibited, and use of dipeptidyl peptidase 4 inhibitors or thiazolidinediones was discouraged. No crossover between treatments was allowed.

Patients did home blood glucose monitoring and were taught about the signs and symptoms of hypoglycaemia. Hypoglycaemia episodes were defined according to the criteria of the American Diabetes Association Workgroup on Hypoglycaemia.?

Cardiovascular events (myocardial infarctions, stroke [including transient ischaemic attacks and ischaemic neurological deficits]) and all deaths that occurred during treatment were adjudicated by a Clinical Endpoint Committee and are part of an ongoing meta-analysis. An independent, masked Pancreatitis Adjudication Committee, comprising three gastrointestinal specialists, adjudicated adverse events suggestive of pancreatitis and all laboratory increases of lipase, amylase, or both that were three or more times the upper limit of normal (appendix pp 8-9). The Pancreatitis Adjudication Committee judged both the probability of events being pancreatitis (definite, probable, possible, or not likely) and the likelihood of association with study drug (definite, probable, possible, or not likely to be related to study medication). For potential events of pancreatitis, medication was withheld and additional diagnostic measures were initiated. Patients were withdrawn from the study upon a diagnosis of pancreatitis. Safety was also monitored by an independent data monitoring committee. Assessment for systemic allergic reactions (appendix p 10) included investigator reporting and standard Medical Dictionary for Regulatory Activities queries for anaphylaxis, angioedema, and severe cutaneous reaction.

The presence of albiglutide antibodies was assessed with a validated enzyme-linked immunosorbent assay (appendix pp 11-12). Confirmed positive samples were tested for albiglutide-neutralising activity in a validated cell-based reporter gene assay.

\section{Outcomes}

The primary endpoint was change in $\mathrm{HbA}_{1 \mathrm{c}}$ from baseline at week 32 for albiglutide versus liraglutide. Secondary endpoints were $\mathrm{HbA}_{1 \mathrm{c}}$ change from baseline over time, change in fasting plasma glucose from baseline over time, the proportion of patients meeting $\mathrm{HbA}_{1 \mathrm{c}}$ treatment goals of below $7 \cdot 0 \%$ and below $6 \cdot 5 \%$, time to hyperglycaemia 
rescue, and change in bodyweight from baseline. Exploratory objectives included the occurrence of hyperglycaemia rescue and treatment satisfaction (assessed in 728 patients from the USA only with the validated Diabetes Medication Satisfaction questionnaire ${ }^{8}$ ). Safety assessments included the occurrence of adverse events, serious adverse events, and death. Safety events of particular interest were pancreatitis, thyroid tumours, systemic allergic reactions, cardiovascular events, gastrointestinal events, hypoglycaemia, and immunogenicity.

\section{Statistical analysis}

With the assumption of an expected treatment difference of $0 \%$ and a standard deviation of $1.1 \%$, the test of albiglutide versus liraglutide would have at least $93 \%$ power with a one-sided, two-sample $t$ test and a test-wise significance level of 0.025 with 320 patients completing the study in each treatment group, for an upper margin of the non-inferiority CI of $0.3 \%$. To allow for an early withdrawal rate of as high as $20 \%$, loss to glycaemic follow-up, and rescue, we randomly allocated at least 400 patients to each treatment group.

The randomised population included all patients randomly assigned to receive study treatment, irrespective of whether or not they actually received it. The modified intention-to-treat population included all randomly assigned patients who received at least one dose of study drug and had a baseline assessment and at least one post-baseline $\mathrm{HbA}_{1 \mathrm{c}}$ assessment. We analysed these patients according to their randomly assigned treatment. The safety population included all randomly assigned patients who received at least one dose of study treatment and were analysed according to treatment received. Imputation for missing observations was applied to efficacy endpoints that were assessed at or before week 32. The last observation carried forward method was used. We did two post-hoc analyses on the per-protocol population, which was defined after database lock and included the subset of the intentionto-treat population that either excluded participants with major protocol violations that were identified before database lock or all participants with any major protocol violation.

We applied the primary analysis of $\mathrm{HbA}_{1 \mathrm{c}}$ change from baseline at week 32 to the modified intention-to-treat sample with an ANCOVA model, with main effects for treatment group, region, history of myocardial infarction, and age, with baseline $\mathrm{HbA}_{1 \mathrm{c}}$ as a continuous covariate. We assessed the difference in treatment effect between the two groups within the ANCOVA model as a leastsquares means contrast, assessed with a one-sided $t$ test at 0.025 significance, with a non-inferiority upper margin of $0.3 \%$ for the change in $\mathrm{HbA}_{1 \mathrm{c}}{ }^{.}$

For patients who received hyperglycaemia rescue therapy, their $\mathrm{HbA}_{1 \mathrm{c}}$ change from baseline (primary endpoint) was censored at the time of rescue. Follow-up assessments continued beyond rescue. Patients who discontinued study participation had their final $\mathrm{HbA}_{1 \mathrm{c}}$ result carried forward for the primary analysis.

We analysed change from baseline over time in $\mathrm{HbA}_{1 \mathrm{c}}$, fasting plasma glucose, and bodyweight analogous to the primary endpoint. We used descriptive statistics to analyse time to meet hyperglycaemia rescue criteria and frequency of attaining $\mathrm{HbA}_{1 \mathrm{c}}$ targets by treatment group, and we assessed inferential treatment comparisons (ie, those with formal statistical hypothesis testing) with nonparametric, covariance-adjusted, extended MantelHaenszel tests. As a supportive analysis, we used a logistic regression model with effects for treatment and other main effects variables to quantify the treatment effects. We analysed time to hyperglycaemia rescue for treatment group differences with pairwise log-rank tests within a Kaplan-Meier model.

Safety analyses included comparative summaries of events. The frequency of adverse events was calculated as the number of participants experiencing the event divided by the total number of participants in each treatment group. We calculated ad-hoc $\mathrm{p}$ values and 95\% CIs with the standard Wald asymptotic method. We compared time to first occurrence of gastrointestinal events with Kaplan-Meier regression and the total number of specific gastrointestinal adverse events with Poisson regression.

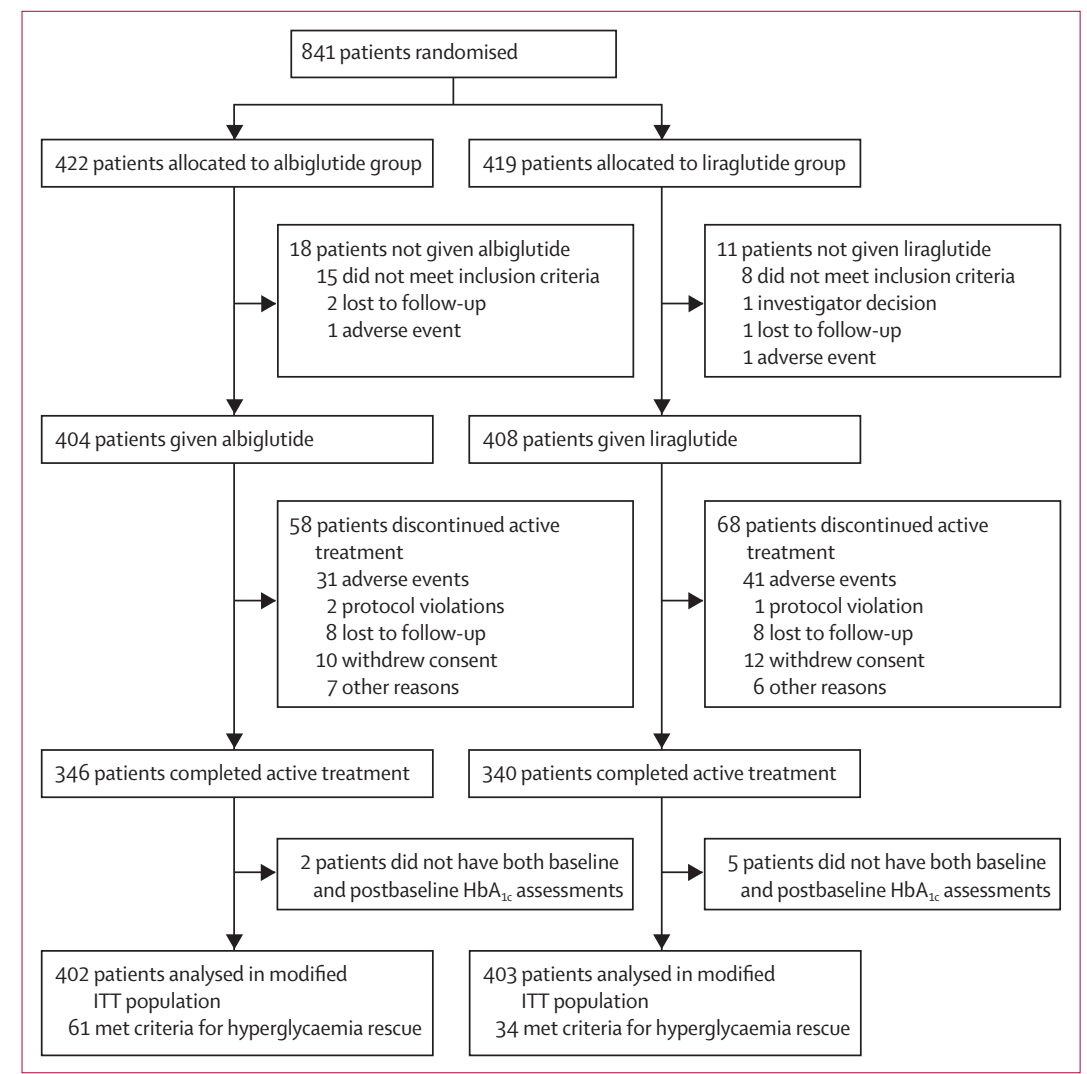

Figure 1: Trial profile

ITT=intention-to-treat. 
All statistical analyses were done with SAS version 9.1. The study is registered with ClinicalTrials.gov, number NCT01128894.

\section{Role of the funding source}

The sponsor of the study participated in the study design, data collection, data review, data analysis, and writing of the report. All authors had full access to all the data in the study. The corresponding author reviewed the trial report (signatory investigator), had full access to all data in the study, and had final responsibility for the decision to submit for publication.

\section{Results}

841 patients with type 2 diabetes were enrolled in this study, and 812 were randomly allocated to the two study drugs (404 albiglutide and 408 liraglutide; figure 1). Table 1 summarises the baseline characteristics of the trial participants, most of whom were already on two or more oral antidiabetic drugs. Mean adherence in the albiglutide

\begin{tabular}{|lcc|}
\hline & $\begin{array}{c}\text { Albiglutide } \\
(\mathbf{n}=404)\end{array}$ & $\begin{array}{c}\text { Liraglutide } \\
(\mathbf{n}=408)\end{array}$ \\
\hline Age (years) & $55 \cdot 4(10)$ & $55 \cdot 8(10)$ \\
\hline Men & $191(47 \%)$ & $218(53 \%)$ \\
Weight $(\mathrm{kg})$ & $91 \cdot 7(21 \cdot 2)$ & $92 \cdot 8(22 \cdot 1)$ \\
\hline BMI (kg/m $\left.{ }^{2}\right)$ & $32 \cdot 8(6 \cdot 0)$ & $32 \cdot 8(5 \cdot 9)$ \\
Previous myocardial infarction & $20(5 \%)$ & $12(3 \%)$ \\
\hline Diabetes duration (years) & $8 \cdot 4(6 \cdot 1)$ & $8 \cdot 3(5 \cdot 6)$ \\
Oral anti-diabetes therapy & & \\
None & 0 & $1(0 \cdot 2 \%)$ \\
Metformin only & $141(35 \%)$ & $149(37 \%)$ \\
Sulfonylurea only & $21(5 \%)$ & $15(4 \%)$ \\
Thiazolidinedione only & $5(1 \%)$ & $3(1 \%)$ \\
Metformin and sulfonylurea & $179(44 \%)$ & $185(45 \%)$ \\
Metformin and thiazolidinedione & $18(5 \%)$ & $23(6 \%)$ \\
Sulfonylurea and thiazolidinedione & $4(1 \%)$ & $6(2 \%)$ \\
Metformin, thiazolidinedione, and & $35(9 \%)$ & $26(6 \%)$ \\
sulfonylurea & & \\
Data are mean (SD) or $n$ (\%). & & \\
\hline Table 1: Baseline characteristics of participants & \\
\hline
\end{tabular}

\begin{tabular}{lcc} 
& $\begin{array}{l}\text { Albiglutide } \\
(\mathbf{n = 4 0 2})\end{array}$ & $\begin{array}{l}\text { Liraglutide } \\
(\mathbf{n = 4 0 3 )}\end{array}$ \\
\hline Baseline $\mathrm{HbA}_{1 \mathrm{cc}}$ & $8.18 \%(0.89)$ & $8.15 \%(0.84)$ \\
\hline Week $32 \mathrm{HbA}_{1 \mathrm{c}}$ & $7.39 \%(1.11)$ & $7.18 \%(1.08)$ \\
\hline $\mathrm{HbA}_{1 \mathrm{c}}$ change from baseline (model-adjusted value*) & $-0.79 \%(-0.78 \%)$ & $-0.98 \%(-0.99 \%)$ \\
\hline Treatment difference for albiglutide vs liraglutide & $0.21 \%(0.08-0.34)$ & $\mathrm{NA}$ \\
\hline Non-inferiority p value for comparison with liraglutide† & 0.0846 &.. \\
\hline Superiority $\mathrm{p}$ value & $\mathrm{NA}$ & $\mathrm{NA}$
\end{tabular}

Data are mean (SD), \% difference, or \% difference $(95 \% \mathrm{Cl})$, unless otherwise indicated. NA=not applicable. *Adjusted for baseline $\mathrm{HbA}_{1 c}$, history of previous myocardial infarction, age group, and region. $\dagger p<0.05$ would indicate non-inferiority.

Table 2: Change in $\mathrm{HbA}_{1 \mathrm{c}}$ from baseline at week 32 for albiglutide versus liraglutide group was greater than $98 \%$, based on returned investigational product single-dose pens; we could not accurately calculate adherence for patients assigned to liraglutide because of the multi-dose pen device used.

Rates of withdrawal from the study were 58/422 $(13 \cdot 7 \%)$ in the albiglutide group and 68/419 (16.2\%) in the liraglutide group (figure 1). The most common reason for discontinuation in both groups was the occurrence of adverse events.

At week 32, $\mathrm{HbA}_{1 \mathrm{c}}$ had decreased significantly from baseline in both groups (table 2). Since the upper bound of the $95 \%$ CI for the treatment difference exceeded the prespecified non-inferiority margin of $0.3 \%$, the criteria for non-inferiority of albigultide were not met. A perprotocol analysis that excluded patients with major protocol violations was consistent with the primary analysis (data not shown).

Subgroup analyses on the primary efficacy endpoint (baseline $\mathrm{HbA}_{1 c}$, sex, race, ethnicity, age, diabetes duration, and background oral antidiabetic drugs) were consistent with the primary endpoint for the overall population (figure 2). Decreases in $\mathrm{HbA}_{1 \mathrm{c}}$ from baseline over time were recorded through to week 32 in both treatment groups, beginning at week 4 (first assessment) and stabilising by week 12 (figure $3 \mathrm{~A}$ ). Changes from baseline over time in fasting plasma glucose were consistent with changes in $\mathrm{HbA}_{1 \mathrm{c}}$. At 32 weeks, least-squares mean change in fasting plasma glucose in the albiglutide group was $-1.22 \mathrm{mmol} / \mathrm{L}$ ( $95 \% \mathrm{CI}-1.45$ to $-1 \cdot 00$ ), and in the liraglutide group was $-1.68 \mathrm{mmol} / \mathrm{L}(-1.91$ to -1.46$)$; treatment difference was $0.46(0 \cdot 14-0 \cdot 78 ; \mathrm{p}=0 \cdot 0048)$ (figure $3 \mathrm{~B})$. The $\mathrm{HbA}_{1 \mathrm{c}}$ treatment goal of lower than $7.0 \%$ was met by 168 of 398 (42\%) patients receiving albiglutide and 208 of $402(52 \%)$ patients receiving liraglutide $(\mathrm{p}=0 \cdot 0023)$, and the goal of $\mathrm{HbA}_{1 \mathrm{c}}$ lower than $6.5 \%$ was met by $78(20 \%)$ patients given albiglutide and $113(28 \%)$ of those given liraglutide $(\mathrm{p}=0 \cdot 0009)$.

Hyperglycaemia rescue criteria occurred in 61/402 $(15 \%)$ of patients in the albiglutide group and 34/403 $(8 \%)$ of those in the liraglutide group by week 32 . The difference in time to hyperglycaemia rescue was statistically significantly in favour of liraglutide $(p=0 \cdot 005)$ and the probability of hyperglycaemia rescue was higher in patients in the albiglutide group from week 12 to 32 (albiglutide vs liraglutide: 0.0286 vs 0.0027 at week 12 ; 0.1333 vs 0.0783 at week 26; and 0.1929 vs 0.1247 at week 32).

Weight loss was significantly greater with liraglutide $(-2 \cdot 19 \mathrm{~kg}, 95 \% \mathrm{CI}-2 \cdot 55$ to $-1 \cdot 83)$ than with albiglutide $(-0.64 \mathrm{~kg},-1.00$ to -0.28$)$, with a treatment difference at 32 weeks of $1.55 \mathrm{~kg}$ (95\% CI 1.05-2.06; p<0.0001). At week 32 , the least-squares mean change in weight from baseline was $-0.6 \mathrm{~kg}$ (SD 3.12) with albiglutide versus $-2 \cdot 2 \mathrm{~kg}(4 \cdot 15)$ with liraglutide. We recorded no meaningful differences in lipid parameters (appendix p 13). 
The most common adverse events were injection-site reactions, gastrointestinal events, and upper respiratory tract infections (table 3). Analysis of injection-site reactions, including the preferred term of injection-site reaction and also several related event terms flagged by the investigator, showed that these reactions occurred more frequently with albiglutide than with liraglutide (albiglutide $12.9 \%$, liraglutide $5.4 \%$; difference between groups $7.5 \%$ [95\% CI 3.6-11.4]; p=0.0002). These reactions resulted in the withdrawal of $11 / 404(2.7 \%)$ patients in the albiglutide group and $1 / 408(0 \cdot 2 \%)$ of those in the liraglutide group. Most events were mild in intensity and lasted for a median of 8-10 days.

Gastrointestinal events were common in both groups (albiglutide 35.9\%, liraglutide 49.0\%; difference between groups $-13 \cdot 1 \%$ [95\% CI $-19 \cdot 9$ to $-6 \cdot 4 \%]$; appendix p 14). Diarrhoea was the most common gastrointestinal event in the abliglutide group and occurred slightly more frequently in this group than the liraglutide group, although this difference was not significant (table 3; appendix p 14). Nausea and vomiting occurred in significantly more patients in the liraglutide group than in the albiglutide group (table 3) - a difference that was most pronounced early in the course of treatment (appendix pp 15-16). 26 of $408(6.4 \%)$ patients withdrew from the study because of gastrointestinal events in the liraglutide group, and ten of $404(2 \cdot 5 \%)$ did so in the albiglutide group.

During the screening phase, roughly $15 \%$ of patients were not eligible to participate because they had a lipase value higher than the upper limit of normal (and about $2 \%$ had a screening lipase value three or more times the upper limit of normal). During the study itself, the Pancreatitis Adjudication Committee undertook masked adjudication of events in 12 patients in the albiglutide group (all lipase elevations) and 13 in the liraglutide group (five gastrointestinal adverse events with or without lipase elevation, and eight cases of raised laboratory lipase values alone). Of these patients, one person in the albiglutide group and two in the liraglutide group were judged to have definite or probable pancreatitis that was at least possibly related to study treatment. Only one of the 25 participantswho was in the liraglutide group and whose event was judged as not likely to be pancreatitis-was admitted to hospital. The remaining cases were adjudicated as possible pancreatitis based on lipase elevation alone (11 albiglutide, eight liraglutide) or unlikely (one liraglutide). No cases of thyroid cancer were diagnosed during our trial. One patient in each group developed a thyroid nodule (both small and neither biopsied). No meaningful changes in calcitonin were recorded in either group.

Investigator-assessed cardiovascular adverse events occurred at a similar rate in the liraglutide group (10.5\%; 59 events) and the albiglutide group (8.2\%; 48 events), treatment difference between groups $-2.4 \%$ (95\% CI $-6 \cdot 4 \%$ to $1 \cdot 6 \%$ ). Hypertension was the most common

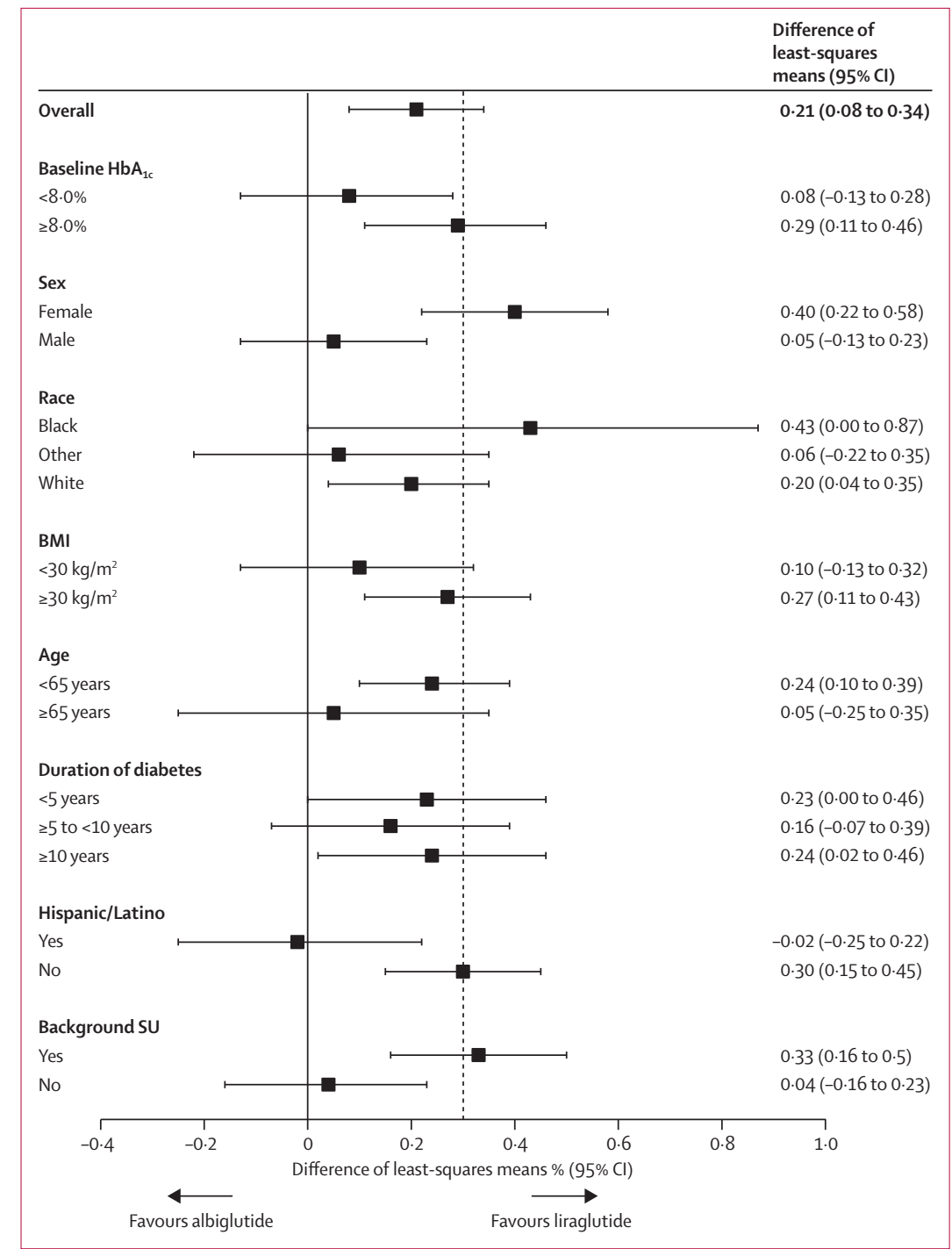

Figure 2: Difference in least-squares means of model-adjusted change from baseline in $\mathrm{HbA}_{1 \mathrm{c}}$ for albiglutide versus liraglutide at week 32 by subgroup category

Analysis is for the modified intention-to-treat population with last observation carried forward. Error bars are $95 \%$ Cls. *Data obtained post hoc

cardiovascular event, with a similar occurrence in both groups.

Following uptitration, a transient increase in heart rate occurred with liraglutide (maximum mean increase at week 4 of 5.7 beats per min), which returned to near baseline during the trial. Patients in the albiglutide group did not have a similar heart rate increase. Mean change in heart rate from baseline to week 32 was 1 beat per min in the albiglutide group and 3 beats per min in the liraglutide group. Mean changes from baseline to week 32 for systolic and diastolic blood pressure were less than $1 \mathrm{mmHg}$ in both groups.

Pre-rescue documented symptomatic hypoglycaemia ( $\leq 3.9 \mathrm{mmol} / \mathrm{L})$ occurred in $10.4 \%$ of patients given albiglutide and $13.0 \%$ of those given liraglutide (difference 


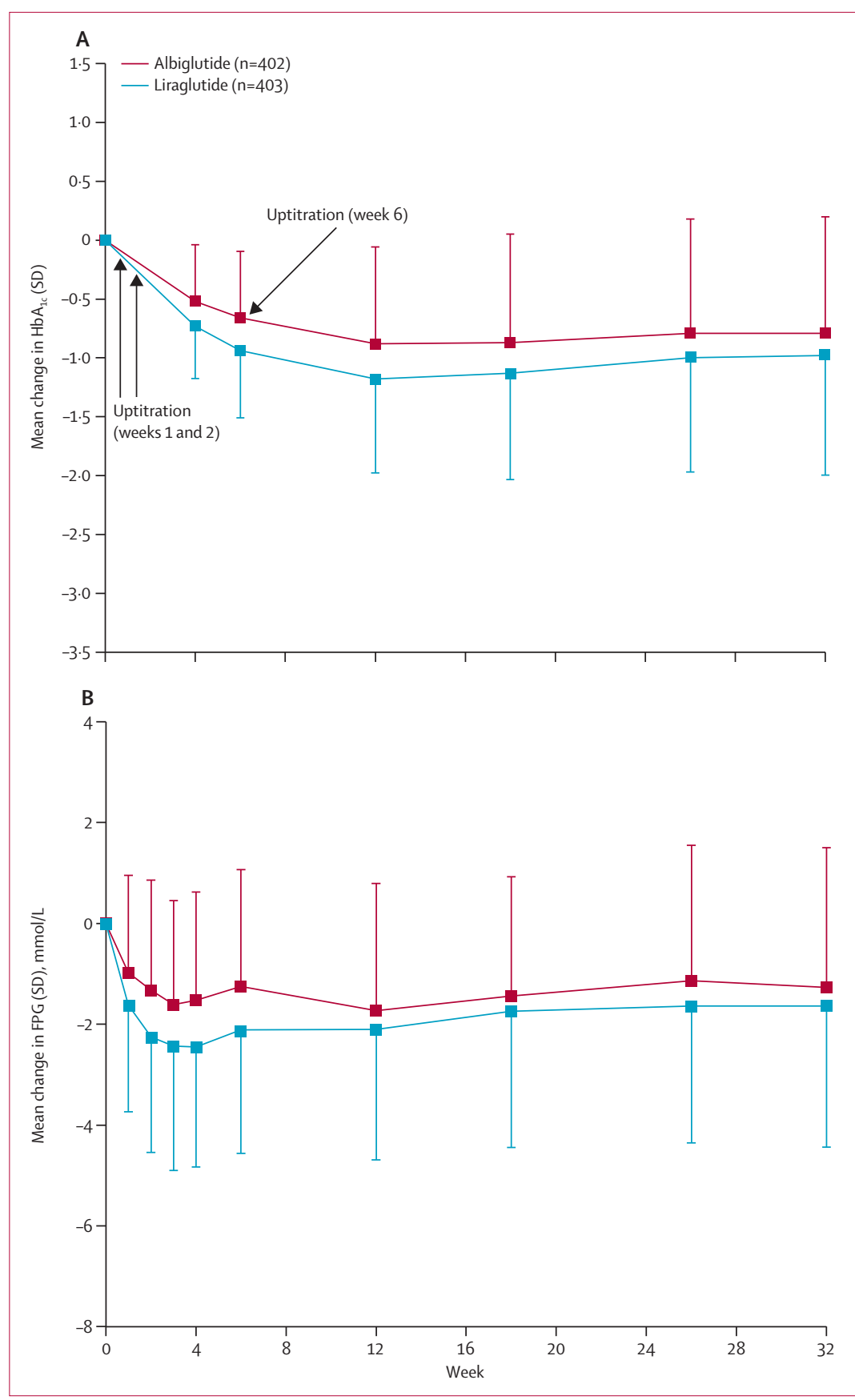

Figure 3: Mean change from baseline through to week 32 in (A) $\mathrm{HbA}_{1 c}$ and (B) fasting plasma glucose Analysis is for the modified intention-to-treat population with last observation carried forward. Error bars are SDs.

between the groups $-2 \cdot 4 \%$; $95 \% \mathrm{CI}-7 \cdot 0$ to $1 \cdot 8 \%$; $\mathrm{p}=0 \cdot 25)$. Most hypoglycaemia events in the albiglutide (>90\%) and liraglutide $(>85 \%)$ groups occurred in patients taking concomitant sulfonylurea therapy. No severe hypoglycaemic events were recorded (appendix p 17).

Albiglutide antibodies developed in 15 of 403 (3.7\%) albiglutide-treated patients (liraglutide antibodies were not assessed); none of the antibodies were neutralising.
Assessment for systemic allergic reaction showed no events of angioedema or anaphylaxis (appendix p 10).

Treatment satisfaction scores, on a scale from 0 (worst) to 100 (best), improved for both treatments at 32 weeks (increase from baseline in mean Diabetes Medication Satisfaction questionnaire of 3.29 for albiglutide [baseline 73.4] and 4.38 for liraglutide [baseline 72.7]; $\mathrm{p}=0 \cdot 207$ for treatment difference).

\section{Discussion}

Head-to-head trials are an important strategy to assess the range of clinical benefits and limitations of different compounds (panel). ${ }^{10-13}$ Our findings show clinically relevant glycaemic lowering with both albiglutide and liraglutide. The predefined statistical outcome for noninferiority of albiglutide of a $95 \%$ CI upper margin of $0 \cdot 3 \%$ was not met. Additionally, both GLP-1 receptor agonists in this study led to weight loss; however, weight loss was less with albiglutide than with liraglutide. Fewer participants in the liraglutide group than in the albiglutide group needed hyperglycaemia rescue. Both treatments led to an increase in treatment satisfaction from baseline. In general, these results are similar to those of DURATION-6, ${ }^{12}$ in which once-weekly exenatide did not meet its prespecified non-inferiority margin compared with liraglutide. Similarly in that trial, more weight loss occurred with liraglutide, but fewer gastrointestinal adverse events occurred with once-weekly exenatide. ${ }^{12}$

In this trial, we also noted differences in the safety and tolerability profiles of the drugs. Injection-site reactions were the most common adverse event with albiglutide and occurred more frequently in patients given albiglutide than in those given liraglutide. Rates of gastrointestinal events overall, especially nausea and vomiting, were lower for albiglutide than liraglutide. Similar to previously reported findings for liraglutide, ${ }^{13-20}$ heart rate increased more after liraglutide uptitration than albiglutide uptitration, and almost returned to normal during continued treatment. Finally, albiglutide and liraglutide showed a low intrinsic rate of hypoglycaemia, since more than $90 \%$ of hypoglycaemia events in the albiglutide group and more than $85 \%$ of those in the liraglutide group occurred in patients on concomitant sulfonylurea treatment.

Asymptomatic elevations of lipase and amylase are common in patients with type 2 diabetes, and in our study roughly $2 \%$ of participants had lipase levels more than three times the upper limit of normal at screening. ${ }^{21}$ A strength of this trial was the fully transparent and very careful masked assessment of any potential case of pancreatitis by three independent gastroenterologists. In this open-label study with routine assessment of pancreatic enzymes, most potential cases were judged as possible pancreatitis based on the prespecified criteria of asymptomatic lipase elevation more than three times the upper limit of normal. The Pancreatitis Adjudication Committee 
identified probable pancreatitis cases on the basis of a combination of gastrointestinal symptoms and lipase elevation that were at least possibly related to study treatment in two liraglutide patients and one albiglutide patient. The symptoms in these three cases did not lead to hospital admission.

In this study, the differences in glycaemic effectiveness, weight reduction, and adverse event profile might be the consequence of the positions of the maximum doses tested on the respective dose-response curves. An alternative explanation is that with a once-weekly GLP-1 receptor agonist, differences in drug exposure might occur throughout the inter-injection interval that affect the efficacy and tolerability profile. The latter explanation would also apply to the head-to-head comparison between once-weekly exenatide and liraglutide. ${ }^{12}$ Finally, albiglutide is a large protein that cannot cross the blood-brain barrier or diffuse into the hypothalamus and area postrema (vomiting centre) as easily as do smaller peptides such as liraglutide. Both efficacy measures and adverse events have been partly linked to centrally mediated effects. ${ }^{22,23}$

This study has several limitations. First, the duration of 32 weeks is quite short in view of the chronic nature of type 2 diabetes. However, this duration is within a range typical of phase 3 diabetes trials. ${ }^{24}$ It allowed for both drugs to come to a steady state, based on their different half-lives, and then for stabilisation of their effects. The albiglutide phase 3 programme includes longer trials (3) years of masked treatment-NCT00838903, NCT00839527, NCT00849056, and NCT00849017) to address whether the effects of albiglutide treatment are sustained for longer periods. A seasonal bias is unlikely to have been introduced into our study since the overall duration of the study conduct was longer than 1 year and the study recruited in both the northern and southern hemispheres. Second, the study was open-label to limit the necessity for sham injections to patients. The primary outcome was a hard endpoint and is unlikely to have been affected by the study design. However, the open-label aspect might have led to more reported events of pancreatitis in liraglutide-treated patients than in those given albiglutide (because liraglutide is an approved drug for which there have been post-marketing reports of pancreatitis), despite similar numbers of patients with raised amylase and lipase levels. This finding was mitigated by the masked review by an endpoints committee.

Most antidiabetes drugs tested in clinical trials reduce $\mathrm{HbA}_{1 \mathrm{c}}$ by $0 \cdot 5-1 \cdot 5 \%$, depending on study design, baseline $\mathrm{HbA}_{1 \mathrm{c}}$ and study population, with combinations resulting in quite similar glycaemic outcomes but offering a range of non-glycaemic effects and attributes. Consistent with the recent position statement of the American Diabetes Association and the European Association for the Study of Diabetes, ${ }^{25}$ the entire clinical picture should be considered so that treatment can be personalised based

\begin{tabular}{|c|c|c|c|c|}
\hline & $\begin{array}{l}\text { Albiglutide } \\
(\mathrm{n}=404)\end{array}$ & $\begin{array}{l}\text { Liraglutide } \\
(\mathrm{n}=408)\end{array}$ & $\%$ difference $(95 \% \mathrm{Cl})$ & pvalue* \\
\hline Any adverse event & $305(75 \cdot 5 \%)$ & $317(77 \cdot 7 \%)$ & $-2.2 \%(-8.0 \%$ to $3.6 \%)$ & 0.4587 \\
\hline Diarrhoea & $60(14.9 \%)$ & $55(13 \cdot 5 \%)$ & $1.4 \%(-3.4 \%$ to $6.2 \%)$ & 0.5752 \\
\hline Upper respiratory tract infection & $42(10 \cdot 4 \%)$ & $45(11 \cdot 0 \%)$ & $-0.6 \%(-4.9 \%$ to $3.6 \%)$ & 0.7705 \\
\hline Nausea & $40(9 \cdot 9 \%)$ & $119(29 \cdot 2 \%)$ & $-19 \cdot 3 \%(-24 \cdot 6 \%$ to $-14 \cdot 0 \%)$ & $<0.0001$ \\
\hline Injection-site reaction $\dagger$ & $28(6 \cdot 9 \%)$ & $5(1 \cdot 2 \%)$ & $5.7 \%(3.0 \%$ to $8 \cdot 4 \%)$ & $<0.0001$ \\
\hline Urinary tract infection & $25(6.2 \%)$ & $23(5 \cdot 6 \%)$ & $0.6 \%(-2.7 \%$ to $3.8 \%)$ & 0.7393 \\
\hline Nasopharyngitis & $24(5 \cdot 9 \%)$ & $28(6 \cdot 9 \%)$ & $-0.9 \%(-4.3 \%$ to $2.4 \%)$ & 0.5914 \\
\hline Increased lipase concentration & $22(5 \cdot 4 \%)$ & $28(6 \cdot 9 \%)$ & $-1 \cdot 4 \%(-4 \cdot 7 \%$ to $1 \cdot 9 \%)$ & 0.4007 \\
\hline Headache & $22(5 \cdot 4 \%)$ & $22(5 \cdot 4 \%)$ & $0.1 \%(-3.1 \%$ to $3.2 \%)$ & 0.9732 \\
\hline Vomiting & $20(5.0 \%)$ & $38(9 \cdot 3 \%)$ & $-4.4 \%(-7.9 \%$ to $-0.8 \%)$ & 0.0154 \\
\hline
\end{tabular}

Data are $\mathrm{n}(\%)$, unless otherwise indicated. ${ }^{*} \mathrm{p}$ values are for the percentage difference. $†$ This analysis includes only events for which injection-site reaction was the preferred term. Additional analyses were done to assess injection-site reactions and other related preferred terms.

Table 3: Adverse events that occurred in $5 \%$ or more of patients in the treatment groups combined.

\section{Panel: Research in context}

\section{Systematic review}

Head-to-head trials allow the efficacy and safety of new drugs to be compared with established treatments. We searched Medline, Embase, and Bios with the following search terms: (((GLP-1 or (GLP NEAR/1 "1") or GLP1 or glucagon NEAR/1 like NEAR/1 peptide NEAR/1 "1") near1 (therap* or agent or agonist)) or exenatide or byetta or byetta NEAR/1 LAR or bydureon or liraglutide or victoza) ANDTI,AB ("vs" OR "versus" OR compar* OR head) AND (clinicaltrials.gov OR "ICTRP" OR eudract OR "NCT*" OR "UTN" or "universal trial number"). No date or language restrictions were used. Our search showed seven randomised trials of at least 28 days duration that compared two glucagon-like peptide-1 (GLP-1) receptor agonists or formulations. In these studies, substantial reductions in $\mathrm{HbA}_{1 \mathrm{c}}$ were reported for all drugs, but differences in efficacy and tolerability between drugs were recorded. No other head-to-head trials have statistically compared albiglutide with another GLP-1 receptor agonist; however, a phase $2 \mathrm{~b}$ study used twice-daily exenatide as a reference group, but no formal statistical analyses were done to compare albiglutide and twice-daily exenatide in this study. ${ }^{5}$

\section{Interpretation}

The results from our study show that both albiglutide and liraglutide treatment led to clinically meaningful reductions in $\mathrm{HbA}_{1 \mathrm{c}}$ at 32 weeks, although albiglutide did not meet the prespecified non-inferiority margin. Additionally, weight loss was significantly greater with liraglutide than with albiglutide. Both treatments were associated with low rates of hypoglycaemia, as expected, but less nausea and vomiting occurred with albiglutide than with liraglutide. These findings indicate that albiglutide could be a suitable alternative to liraglutide for some patients with type 2 diabetes who are candidates for GLP-1 receptor agonist treatment. Our study is the first to make statistical comparisons between albiglutide and another GLP-1 receptor agonist.

not only $\mathrm{HbA}_{1 \mathrm{c}}$ reduction reported in many trials, but also on tolerability, safety, and frequency and ease of administration.

\section{Contributors}

All authors fulfil International Committee of Medical Journal Editors criteria for authorship. All authors contributed to the interpretation of the data and the development and approval of the report. All authors were fully involved in the development of this Article and assume responsibility for the direction and content. 
Harmony 7 Study Investigators

K Adamson, A Ahmann, C W Ahn, D Ajani, L Akright, L Alwine, O Alzohaili, N Andrawis, H Arbañil Huaman, S Arora, T Bailey, A Barnett, M Baron, L Barreda Caceres, J Barrera, J Berg, R Bertenshaw, B Bode, D Bolton, M Brito, S Brock, A Brockmyre, R Broker, O Brusco Jr, R Buynak, R Canadas-Zizzias, G Canas, J Capo, M Castillo Gamarra, H Cathcart, E A Catindig, S Chilka, YW Cho, D S Choi, L Chuck, M Cooper, C Corder, H Cruz, K Darzy, R de la Rosa, L De Teresa, R DeGarmo, M D'Emden, M Denopol, M DiGiovanna, D Escalante, F Fakih, N Farris, M Feinglos, R Fillmore, D Fitz-Patrick, A Forker, G Fulcher, N Gabra, J Gabriel, M Gerstman, L Glaser, L Gnudi, L Gonzales Bravo, E Gonzalez, R Graf, M Greenwald, G Grunberger, I Harman-Boehm, T Herskovits, A Higgins, J Hoekstra, P Hollander, E Hubach, D Huffman, R Ison, H C Jang, C Jimeno, S Jones, J Karl, S Kemp, C Ketels, M Kipnes, C Knopke, E Kolettis, A Lacour, G Ledesma, D Lee, G Lefebvre, D Liljenquist, R Lipetz, M Look, K Lucas,

A Luna Figueroa, L Maletz, I Marar, R Mathur, S Mayeda, M McCartney, J McGill, D Metz, D Mihalyi, K W Min, O Minuchin, R Mirasol, D Nelson, C Nolan, P Norwood, M Oberoi, F Ovalle, D Pace, M Pace, J Pappas, N Patel, A Patron, S Powell, R Pratley, J Proietto, M Rendell, A Roberts, R Rosen, P Rosenblit, J Rosenstock, J Rothman, G Ruoff, A Russell, B Samuels, D Sanderlin, R Sanders, J Schmidt, J L Selam, R Severance, M Sewell, S Shachar, R Simpson, T Smith, J Solis Villanueva, A Soto, J Sparks, J Stephens, R Stewart, D Streja, R Strzinek, R A Sy, S Teniola, R Tidman, H Toro, R Upender, J R Urgeles Planella, M Valitutto, V Vishlitzky, R Wade, J Wahle, P Weissman, D Williams, C Yao, M D L Ygpuara, D Yue, L Zapata Rincón.

\section{Members of the Pancreatic Aadjudication Committee}

Firas Al-Kawas (Chairman; Georgetown University, Washington, DC, USA); Michelle Anderson (University of Michigan, MI, USA); and Robert Enns (University of British Columbia, BC, Canada).

\section{Conflicts of interest}

REP has served as a consultant or speaker for, and/or has received grants, research support, or honoraria from, Novartis, Lilly, Eisai, Takeda, Novo Nordisk, Merck, Mannkind, AstraZeneca/Bristol-Myers Squibb, Roche, Sanofi-Aventis, GlaxoSmithKline, Pfizer, and Lexicon. All honoraria from these activities are directed to a non-profit foundation. JR has served on scientific advisory boards for, and has received honoraria, consulting fees, grants, or research support from, GLP-1 receptor agonist manufacturers: GSK, Novo Nordisk, Sanofi, Eli Lilly, Roche, Amylin, and Intarcia. All honoraria from these activities are directed to a non-profit foundation. MAN has served on scientific advisory boards for, and has received honoraria, consulting fees, grants or research support from, Berlin Chemie, Lilly, Merck Sharp \& Dohme, Novartis, AstraZeneca, Boehringer Ingelheim, GlaxoSmithKline, MetaCure, Roche, Novo Nordisk, Tolerx, Amylin, Bristol-Myers Squibb, Diartis, F Hoffmann-La Roche, Intarcia, MannKind, Sanofi-Aventis, Takeda, Versartis, and Wyeth. AHB has served on scientific advisory boards for, and has received honoraria, consulting fees, grants, or research support from, GLP-1 receptor agonist manufacturers, GSK, Novo Nordisk, Eli Lilly, Roche, and Sanofi-Aventis. MNF has received research support and has served as a consultant for GlaxoSmithKline. FO has served on scientific advisory boards for, and has received consulting fees and grant support from, Novo Nordisk, Janssen, Medtronic, Boehringer-Ingelheim, Bristol-Myers Squibb, and Sanofi-Aventis. IH-B has served on scientific advisory boards for and received honoraria or consulting fees support from GLP-1 receptor agonist manufacturers, GlaxoSmithKline, Novo Nordisk, Eli Lilly, and Sanofi-Aventis. SJ, MS, RS, and JY are employed by and shareholders of GlaxoSmithKline. We declare that we have no other conflicts of interest.

\section{Acknowledgments}

GlaxoSmithKline provided funding for this study. Some data from this study were presented at the annual meetings of the American Diabetes Association in 2012 (poster 945-P) and 2013 (poster 1010-P). We thank the following employees of GlaxoSmithKline for their specific contributions: Caroline Perry for study management support, Alan Martin for management and oversight of the treatment satisfaction component within this study, and Sergio Forero-Schwanhaeuser and Douglas L Wicks for management of manuscript development. Editorial support also was provided by Beth Burke (assistance with the production of draft outline, production of the first draft, assembly of tables and figures, and collation of author comments), Joelle Suchy (assistance with the production of draft outline, production of the first draft, assembly of tables and figures, collation of author comments, fact checking, and referencing), and Tammie Anderson (MediTech Media, Hamilton, NJ, USA) for editorial project management, which was funded by GlaxoSmithKline.

\section{References}

1 Drucker DJ, Nauck MA. The incretin system: glucagon-like peptide- 1 receptor agonists and dipeptidyl peptidase- 4 inhibitors in type 2 diabetes. Lancet 2006; 368: 1696-05.

2 Garber AJ. Long-acting glucagon-like peptide 1 receptor agonists: a review of their efficacy and tolerability. Diabetes Care 2011 34 (suppl 2): S279-84.

3 Victoza (package insert). http://www.novo-pi.com/victoza.pdf (accessed Jan 17, 2014).

4 Matthews JE, Stewart MW, De Boever EH, et al. Pharmacodynamics, pharmacokinetics, safety, and tolerability of albiglutide, a long-acting glucagon-like peptide-1 mimetic, in patients with type 2 diabetes. J Clin Endocrinol Metab 2008; 93: 4810-17.

5 Rosenstock J, Reusch J, Bush M, Yang F, Stewart M, Albiglutide Study Group. Potential of albiglutide, a long-acting GLP-1 receptor agonist, in type 2 diabetes: a randomized controlled trial exploring weekly, biweekly, and monthly dosing. Diabetes Care 2009; 32: $1880-86$

6 Rosenstock J, Stewart MW. Albiglutide. Drugs Fut 2010; 35: 701-12.

7 Bush MA, Matthews JE, De Boever EH, et al. Safety, tolerability, pharmacodynamics and pharmacokinetics of albiglutide, a longacting glucagon-like peptide-1 mimetic, in healthy subjects. Diabetes Obes Metab 2009; 11: 498-505.

8 Brod M, Skovlund SE, Wittrup-Jensen KU. Measuring the impact of diabetes through patient report of treatment satisfaction, productivity and symptom experience. Qual Life Res 2006; 15: 481-91.

9 US Department of Health and Human Services, Food and Drug Administration, Center for Drug Evaluation and Research (CDER), and Center for Biologics Evaluation and Research (CBER). Guidance for industry. Non-inferiority clinical trials. March, 2010. http://www.fda.gov/downloads/Drugs/.../Guidances/UCM202140. pdf (accessed Jan 20, 2014).

10 Blevins T, Pullman J, Malloy J, et al. DURATION-5: exenatide once weekly resulted in greater improvements in glycemic control compared with exenatide twice daily in patients with type 2 diabetes. J Clin Endocrinol Metab 2011; 96: 1301-10.

11 Drucker DJ, Buse JB, Taylor K, et al. Exenatide once weekly versus twice daily for the treatment of type 2 diabetes: a randomised, open-label, non-inferiority study. Lancet 2008; 372: $1240-50$.

12 Buse JB, Nauck M, Forst T, et al. Exenatide once weekly versus liraglutide once daily in patients with type 2 diabetes (DURATION-6): a randomised, open-label study. Lancet 2013; 381: 117-24.

13 Buse JB, Rosenstock J, Sesti G, et al. Liraglutide once a day versus exenatide twice a day for type 2 diabetes: a 26-week randomised parallel-group, multinational, open-label trial (LEAD-6). Lancet 2009; 374: 39-47.

14 Garber A, Henry R, Ratner R, et al. Liraglutide versus glimepiride monotherapy for type 2 diabetes (LEAD-3 mono): a randomised, 52-week, phase III, double-blind, parallel-treatment trial. Lancet 2009; 373: 473-81.

15 Marre M, Shaw J, Brandle M, et al. Liraglutide, a once-daily human GLP-1 analogue, added to a sulphonylurea over 26 weeks produces greater improvements in glycaemic and weight control compared with adding rosiglitazone or placebo in subjects with type 2 diabetes (LEAD-1 SU). Diabet Med 2009; 26: 268-78.

16 Nauck M, Frid A, Hermansen K, et al. Efficacy and safety comparison of liraglutide, glimepiride, and placebo, all in combination with metformin, in type 2 diabetes: the LEAD (Liraglutide Effect and Action in Diabetes)-2 study. Diabetes Care 2009; 32: 84-90.

17 Russell-Jones D, Vaag A, Schmitz O, et al. Liraglutide vs insulin glargine and placebo in combination with metformin and sulfonylurea therapy in type 2 diabetes mellitus (LEAD-5 met+SU): a randomised controlled trial. Diabetologia 2009; 52: 2046-55. 
Articles

18 Zinman B, Gerich J, Buse JB, et al. Efficacy and safety of the human glucagon-like peptide-1 analog liraglutide in combination with metformin and thiazolidinedione in patients with type 2 diabetes (LEAD-4 Met+TZD). Diabetes Care 2009; 32: 1224-30.

19 Pratley R, Nauck M, Bailey T, et al. One year of liraglutide treatment offers sustained and more effective glycaemic control and weight reduction compared with sitagliptin, both in combination with metformin, in patients with type 2 diabetes: a randomised, parallel-group, open-label trial. Int J Clin Pract 2011; 65: 397-407.

20 Mundil D, Cameron-Vendrig A, Husain M. GLP-1 receptor agonists: a clinical perspective on cardiovascular effects. Diab Vasc Dis Res 2012; 9: 95-108.

21 Malloy J, Gurney K, Shan K, Yan P, Chen S. Increased variability and abnormalities in pancreatic enzyme concentrations in otherwise asymptomatic subjects with type 2 diabetes. Diabetes Metab Syndr Obes 2012; 5: 419-24.
22 Hayes MR. Neuronal and intracellular signaling pathways mediating GLP-1 energy balance and glycemic effects. Physiol Behav 2012; 106: 413-16.

23 Cabou C, Campistron G, Marsollier N, et al. Brain glucagon-like peptide-1 regulates arterial blood flow, heart rate, and insulin sensitivity. Diabetes 2008; 57: 2577-87.

24 US Department of Health and Human Services, Food and Drug Administration, Center for Drug Evaluation and Research. Guidance for Industry. Diabetes mellitus: developing drugs and therapeutic biologics for treatment and prevention. February, 2008. http://www.fda.gov/downloads/Drugs/Guidances/ucm071624.pdf (accessed Jan 28, 2014).

25 Inzucchi SE, Bergenstal RM, Buse JB, et al. Management of hyperglycaemia in type 2 diabetes: a patient-centered approach: position statement of the American Diabetes Association (ADA) and the European Association for the Study of Diabetes (EASD). Diabetologia 2012; 55: 1577-96. 